\title{
Letter to the Editor \\ The Challenges of Patients With Epilepsy During \\ Coronavirus 2019 Epidemic
}

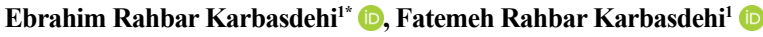

1. Department of Psychology, Faculty of Literature and Humanities, University of Guilan, Rasht, Iran.

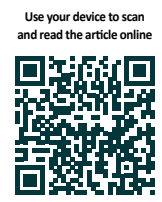

Crtation Rahbar Karbasdehi E, Rahbar Karbasdehi F. The Challenges of Patients With Epilepsy During Coronavirus 2019 Epidemic. Journal of Research \& Health. 2021; 11(6):363-364. http://dx.doi.org/10.32598/JRH.11.6.1927.1

doijhttp://dx.doi.org/10.32598/JRH.11.6.1927.1

\section{Dear Editor}

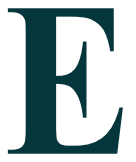

pilepsy is a disease characterized by a spontaneous recurrence of unprovoked seizures and is one of the most common chronic neurological diseases [1]. The prevalence of this disease in Iran is higher than international standards. Currently, about $1 \%$ to $1.5 \%$ of people in Iran have epilepsy, which indicates that these patients in Iran are almost three times more than in Europe [2].

Coronavirus 2019 (COVID-19) is a type of viral pneumonia that broke out in December 2019 in Wuhan, China, and is now spreading rapidly worldwide. The Centers for Disease Control and Prevention states that COVID-19 is a risk factor for neurological diseases, including epilepsy. However, some underlying conditions (e.g., smoking, obesity, diabetes, heart disease, lung disease, and cancer) are risk factors, too [3]. Hence, epilepsy patients with other comorbidities may be more likely to catch COVID-19. CDC (The Centers for Disease Control and Prevention) has already suggested COVID-19 protection measures. So anyone, including people with epilepsy, can use these measures.

COVID-19 can target the central nervous system because two angiotensin-converting enzyme receptors have been identified at the membranes of neurons and glial cells [4]. The reported symptoms of COVID-19 are mainly related to respiratory or gastrointestinal systems. Usually, epilepsy patients during COVID-19 or any other infectious disease may have a fever that leads to seizures [5]. In addition, the relationship between antiepileptic drugs and prescription drugs for COVID-19 needs further investigation.

At present, no specific drug has been approved for $\mathrm{CO}$ VID-19, and certain compounds are not recommended to prevent drug interactions in certain diseases, and this issue needs more attention. Levetiracetam is one of the drugs that does not interact with any drugs. Drug interactions should be considered when introducing or prescribing antiepileptic drugs. In addition, some medications, such as antihistamines, lower the seizure threshold. Some epilepsy drugs can affect the immune system, including everolimus and steroids, which are used to treat tuberous sclerosis complex and autoimmune epilepsy, respectively. However, according to some studies, everolimus can prevent viral infections.

Meanwhile, corticosteroids are related to the risk of infectious diseases [6]. Hence, drugs should be selected individually in clinical settings. It is also essential to inform patients in clinical settings that they should not discontinue antiepileptic medications without the advice of a physician, even if it may affect the immune system [7].

\section{* Corresponding Author:}

Ebrahim Rahbar Karbasdehi, MA.

Address: Department of Psychology, Faculty of Literature and Humanities, University of Guilan, Rasht, Iran.

Phone: +98 (911) 8057926

E-mail: ebrahim.rahbar74@gmail.com 
There are significant problems in patients with epilepsy that can exacerbate COVID-19 conditions. For example, recurrent attacks of epilepsy cause malnutrition, and nutritional status is related to the immune system, and going to the emergency room due to excessive or uncontrollable seizures can expose the patient to the COVID-19. However, there is a shortage of mechanical ventilators in clinical settings in regions with high levels of COVID19 patients. Therefore, in these conditions, seizure control, as well as prevention of COVID-19, is crucial [8].

However, seeing physicians and periodic visits of patients with epilepsy can put them in crowded situations. This situation can be reduced by introducing telemedicine and purchasing prescription drugs lasting a minimum of a few months. Telemedicine makes it possible to consult a physician without facing crowded situations and thus prevent the spread of COVID-19 among patients. Hence, more attention should be paid to the introduction and implementation of telemedicine [6].

Intractable epilepsy can be evaluated and diagnosed through video-encephalogram monitoring and hospitalization. Surgical treatments for epilepsy may be delayed to prevent the further spread of the COVID-19 between medical personnel and patients. Most hospitals are prepared for COVID-19 patients who need intensive care. Patients with epilepsy who need immediate intervention should follow the COVID-19 protection protocol [9].

Providing this information to people with epilepsy and their families is very important to prevent them from becoming infected with the COVID-19. In addition, spreading the correct information reduces unnecessary anxiety and stress. Educating patients with epilepsy can reduce the frequency of seizures and accidental trauma caused by seizures. However, the Internet and social media may be unofficial, unreliable, or misleading sources of information, which may cause unnecessary panic among people. A system needs to be set up to disseminate credible sources of information, and physicians must share information, continue research, and share known facts with patients with epilepsy and their families.

\section{Ethical Considerations}

\section{Compliance with ethical guidelines}

There were no ethical considerations to be considered in this research.

\section{Funding}

This research did not receive any grant from funding agencies in the public, commercial, or non-profit sectors.

\section{Authors' contributions}

Both authors equally contributed to preparing this article.

\section{Conflict of interest}

The authors declared no conflict of interest.

\section{References}

[1] Fiest KM, Sauro KM, WiebeS, PattenSB, Kwon CS, Dykeman $\mathrm{J}$, et al. Prevalence and incidence of epilepsy: A systematic review and meta-analysis of international studies. Neurology. 2017; 88(3):296-303. [DOI:10.1212/WNL.0000000000003509] [PMID] [PMCID]

[2] Asadi-Pooya AA, Simani L. [Epilepsy syndromes in Iran: A systematic review (Persian)]. Acta Neurol ogica Scandinavica. 2021; 143(5):475-80. [DOI:10.1111/ane.13381] [PMID]

[3] Jordan RE, Adab P, Cheng KK. Covid-19: Risk factors for severe disease and death. The BMJ. 2020; 368:m1198. [DOI:10.1136/bmj.m1198] [PMID]

[4] Baig AM, Khaleeq A, Ali U, Syeda H. Evidence of the covid-19 virus targeting the CNS: Tissue distribution, host-virus interaction and proposed neurotropic mechanisms. ACS Chemical Neuroscience. 2020; 11(7):995-8. [DOI:10.1021/ acschemneuro.0c00122] [PMID] [PMCID]

[5] Li Z, Liu T, Yang N, Han D, Mi X, Li Y, et al. Neurological manifestations of patients with covid-19: Potential routes of SARS-CoV-2 neuroinvasion from the periphery to the brain. Frontiers in Medicine. 2020; 14(5):533-41. [DOI:10.1007/ s11684-020-0786-5] [PMID] [PMCID]

[6] Kuroda N. Epilepsy and covid-19: Associations and important considerations. Epilepsy \& Behavior. 2020; 108:107122. [DOI:10.1016/j.yebeh.2020.107122] [PMID] [PMCID]

[7] Andersen ML, Tufik S, Colombo AL, Cavalheiro EA, Cysneiros RM, Scorza FA. Sudden unexpected death in children with epilepsy: The many faces of fungal pathogenicity. Medical Hypotheses. 2012; 79(2):127-8. [DOI:10.1016/j.mehy.2012.03.015] [PMID]

[8] Truog RD, Mitchell C, Daley GQ. The toughest triage-allocating ventilators in a pandemic. The New England Journal of Medicine. 2020; 382(21):1973-75. [DOI:10.1056/NEJMp2005689] [PMID]

[9] Iacobucci G. Covid-19: All non-urgent elective surgery is suspended for at least three months in England. The BMJ. 2020; 368:m1106. [DOI:10.1136/bmj.m1106] [PMID] 